\title{
Ophthalmic artery occlusion following lateral orbitotomy
}

\author{
Swathi A. R. ${ }^{1}$, Mahesh P. Shanmugham², Saptagirish Rambhatla ${ }^{3, *}$ \\ 1Fellow, 2,3HOD, 1,3Dept. of Orbit and Oculoplasty, 2Dept. of Vitreoretina and Ocular Oncology, Sankara Eye Hospital, \\ Marathahalli, Bengaluru, Karnataka, India
}

*Corresponding Author:

Email: saptagirishr@gmail.com

\begin{abstract}
Visual loss following orbital surgery, though rare, is a devastating complication. Ophthalmic artery occlusion as a cause is even more rare and grave as the vision loss is irreversible. We report one such case following lateral orbitotomy for an orbital schwannoma in a young man who denied light perception post operatively and was diagnosed to have an ophthalmic artery occlusion.
\end{abstract}

Keywords: Schwannoma, Orbitotomy, Vision loss, Ophthalmic artery occlusion.

\section{Introduction}

Schwannomas account for $1 \%$ of orbital tumours. Lateral orbitotomy has been the preferred approach for retroconal tumours as it offers a wide operational field. Post operative blindness following the surgery can be catastrophic. The authors report a rare case of unexpected visual loss following an orbital surgery.

\section{Case History}

A thirty six year old man visited our hospital with complaints of prominence of left eye since fourteen years. His best corrected visual acuity was 6/9 in the right eye and $6 / 6$ in his left eye. Anterior and posterior segment examination of the right eye was normal. Left eye showed an axial proptosis with increased resistance to retropulsion [Fig. 1a]. Pupillary reaction was brisk. Orbital rim was intact, extraocular movements were normal, no regional lymph nodes palpable. His color vision and full threshold visual fields were normal. CT scan suggested a large, well defined intraconal mass in the left orbit between the lateral rectus and the optic nerve extending from the mid orbit extending till the orbital apex [Fig. 1b]. A provisional diagnosis of cavernous hemangioma or schwannoma was made. Left lateral orbitotomy under guarded prognosis was planned after a detailed discussion of the possible complications with the patient.
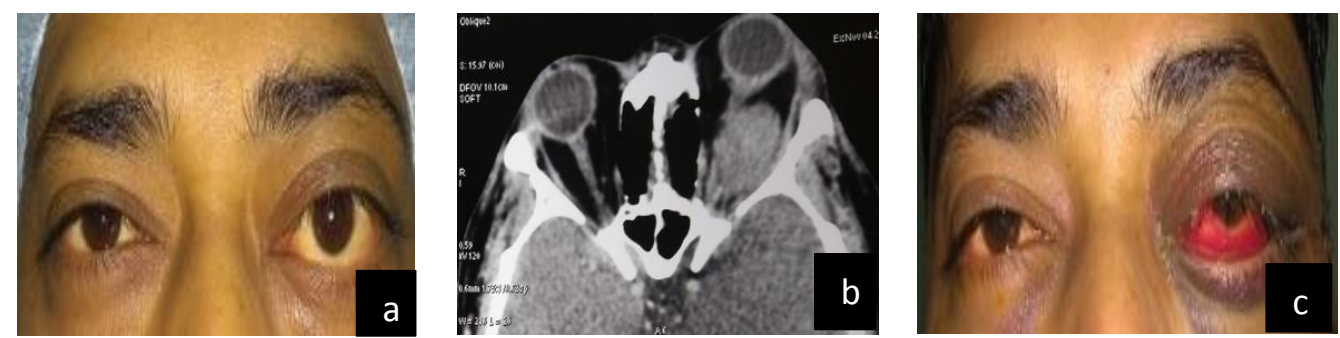

Fig. 1: a:Clinical photograph showing proptosis; b: CT scan, axial view showing an intraconal mass extending upto apex; c: Postoperative clinical photograph showing proptosis and ecchymosis

A lateral orbitotomy with a Berke's incision was perfomed. Intraoperatively, a large firm mass, abutting the optic nerve was noted. Blunt dissection was done and the mass was removed in toto. A sudden dilatation of pupil was noted following removal of the tumour. . Intravenous dexamethasone (8mg in $2 \mathrm{ml}$ ) was given immediately. On the first post-operative day, patient denied perception of light. His extra ocular movements were restricted and mydriasis was noted [Fig. 1c]. The drain placed intraoperatively had a collection of $4 \mathrm{cc}$ of blood. He was given intravenous methylprednisolone of $1 \mathrm{~g}$ for three consecutive days. On the second day, his fundus showed arteriolar attenuation with retinal edema. His intraocular pressures were $13 \mathrm{mmHg}$. Ocular massage and anterior chamber paracentesis was done and the pressures were brought down to $9 \mathrm{mmHg}$. The continued denial of perception of light and absence of a cherry red spot on the retina raised a suspicion of ophthalmic artery occlusion [Fig. 2a, b]. An orbital MRI venography and CT angiography was done to rule out any flow abnormalities in the left orbit which showed non-perfusion of left ophthalmic artery probably due to thrombosis. Patient was referred to an intervention radiologist for a possible thrombolysis. However, the radiologist did not want to intervene as no embolus was noted on MRA. On the fifth postoperative day, there was a slight resolution of retinal edema but the patient still denied light perception. Histopathology suggested features of schwannoma [Fig. 2c]. Patient was explained about the nil visual prognosis in his left eye and the need for a regular follow up for his right eye. 

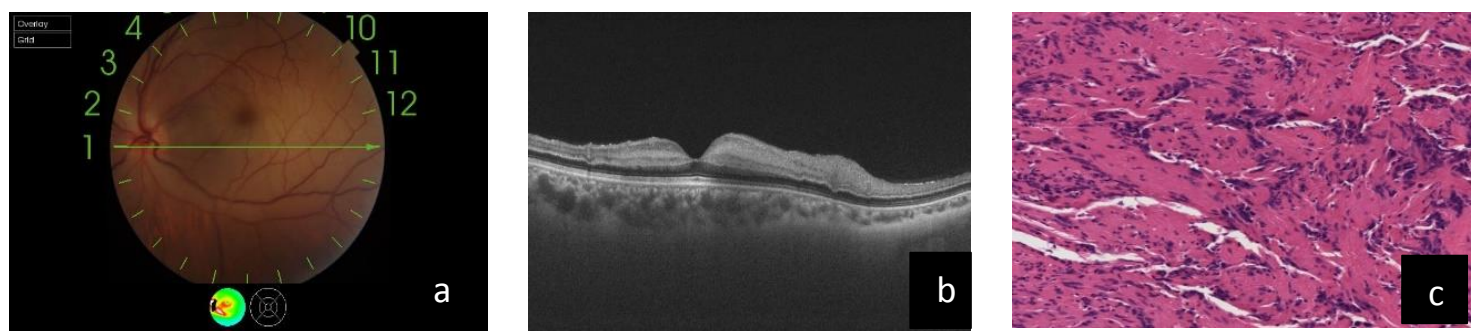

Fig. 2: a: Fundus photograph of left eye showing retinal edema with absence of cherry red spot; b: OCT showing disorganized and hyper reflective retinal layers with increased retinal thickness; c: microphotograph showing typical features of schwannoma

\section{Discussion}

Kronlein ${ }^{1}$ in 1889 was the first person to describe the lateral approach for orbital lesion. This was later modified by Berke ${ }^{2}$ in 1953 which was further modified in 1976 by Maroon and Kennerdell. ${ }^{3}$ This approach offers a better access to larger tumors extending upto the apex and hence was our choice for the case.

There is a striking resemblance of our case with a similar case reported by Anasua Ganguly et $\mathrm{al}^{4}$ where a transconjunctival orbitotomy performed for orbital schwannoma resulted in post operative blindness. In both the cases, the presentation, location and histopathological features of the tumour are similar. The clinical course has also been similar.

Visual loss is a potential severe complication of orbital surgery and its incidence has been estimated to be $0.2 \%$ by Bonavolonta ${ }^{5}$ Various putative mechanisms have been put forth to explain the visual loss.

Harris and Perez $z^{6}$ found that small orbital tumors in the medial orbit are extracted more easily than large tumors as their removal is troubled by tighter tissue adhesions, reduced working room and limited posterior view. Large tumours might be expected to increase the risk of postoperative blindness for two reasons: first, large tumours jeopardize preoperative optic nerve function, especially where the optic nerve is compressed at the orbital apex, and, secondly, because the removal of large tumours requires more surgical manipulation. As our patient had a large tumour that was abutting the optic nerve, he was at a higher risk to develop complications.

Another possible mechanism could have been a post operative vasospasm due to accumulation of inflammatory mediators in the orbital apex resulting in posterior ischemic optic neuropathy which is more frequently noted in surgeries of tumors located in the apical region as this region receives its blood supply solely from perforating dural vessels. ${ }^{7}$ Some blood flow is re-established as vasospasm reverses, but not before there might have been critical level of ischaemia and irreversible loss of optic nerve function.
Post operative hematoma was ruled out in the post operative CT scans.

Extraocular motility restriction can be explained as a transient third nerve palsy due to the surgical dissection in the orbital apex. Mydriasis following tumour removal can result due to injury to the optic nerve or as consequence of the surgical trauma of the ciliary nerves located on the lateral aspect of the optic nerve.

\section{Conclusion}

To conclude, this case report emphasizes the need to further research towards discerning the cause of individual susceptibility so that we may identify and inform those who are at risk before surgery.

Funding: No funding sources.

Conflict of interest: None declared.

\section{References}

1. Krönlein RU. Zur pathologie and operative Behandlung der dermoidcysten der orbita. Beitr Klin Chir 1889;4:149-63.

2. Berke RN. A modified Krönlein operation. Trans Am Opthalmol Soc 1953;51:193-231.

3. Maroon JC, Kennerdell JS. Lateral microsurgical approach to intraorbital tumors. J Neurosurg 1976;44:556-61.

4. Ganguly A, Pappuru RR, Ali MJ, Mishra DK, Naik MN. Ophthalmic artery occlusion following transconjunctival orbitotomy. Ophthalmic Plastic and Reconstructive Surg 2017;33(3):S171-3.

5. Bonavolonta` G. Postoperative blindness following orbital surgery. Orbit 2005;24:195-200.

6. Harris GJ, Perez N. Surgical sectors of the orbit: using the lower fornix approach for large, medial intraconal tumors. Ophthal Plast Reconstr Surg 2002;18:349-54.

7. Buono LM, Foroozan R: Perioperative posterior ischemic optic neuropathy: Review of the literature. Surv Ophthalmol 2005;50:15-26.

How to cite the article: A. Swathi, Shanmugham M.,
Rambhatla S.Ophthalmic artery occlusion following
lateral orbitotomy. Int J Ocul Oncol Oculoplasty
$2018 ; 4(3): 146-147$.

